\title{
Five-Year Prospective Study of Immediate/Early Loading of Fixed Prostheses in Completely Edentulous Jaws with a Bone Quality-Based Implant System
}

\author{
Carl E. Misch, BS, DDS, MDS;* Marco Degidi, $\mathrm{MD}^{\dagger}$
}

\begin{abstract}
Background: The concept of immediate loading of root-form implants for fixed restorations has received increasing interest over the last 5 years. Several authors have commented on parameters that may influence results, including implant number, implant length, bone density, and patient habits. The trigger for bone remodeling around an implant may occur from the surgical trauma of insertion or the mechanical environment of strain at the interface. In the classic two-stage approach, these were divided episodes, separated by 3 to 6 months. Immediate loading compresses this time frame; the two driving mechanisms for bone repair occur concurrently. A scientific approach to the interface development is to match the bone healing response of trauma (woven bone of repair) to the response of mechanical load (reactive woven bone), so the sum of these two entities does not result in fibrous tissue formation and clinical mobility of the implant.
\end{abstract}

Purpose: It is the purpose of this article to review the scientific rationale of these statements and coordinate them to bone physiology and bone biomechanics.

Materials and Methods: Findings from previous reports in the literature were reviewed and summarized to form the basis of a prospective study using a bone quality-based implant system (Biohorizons ${ }^{\circledR}$, Maestro ${ }^{\mathrm{TM}}$ Dental Implants, Birmingham, AL, USA). A transitional prosthesis was delivered either on the day of surgery or within 2 weeks for 30 patients and 31 arches. A total of 244 implants were used to support these restorations, for an average of 7.8 implants per prosthesis. After 4 to 7 months, the final restorations were fabricated. One year after the final restoration was loaded, the implant survival was $100 \%$; the 31 restorations also had a survival of $100 \%$ over this time frame. This report presents these implants and restorations over a 1 - to 5 -year period, with an average follow-up period of 2.6 years.

Results: The bone loss from implant insertion to final prosthesis delivery averaged $0.7 \mathrm{~mm}$. The first-year bone loss after final prosthesis delivery averaged $0.07 \mathrm{~mm}$. A slight increase in bone height was observed after the first year, but generally no increase was observed over the remaining evaluation period.

Conclusions: In the current report, no implant failure occurred, and crestal bone loss values were similar to or less than values reported with the conditional two-stage approach. This may be related to the number and position of implants, implant design, and/or the surface condition of the implant loading.

KEY WORDS: dental implant, clinical study, immediate load, early load

\footnotetext{
*Adjunct associate professor, Department of Periodontics/Prevention/ Geriatrics, School of Dentistry, University of Michigan, Ann Arbor, $\mathrm{MI}$; private practice, Birmingham, MI, USA; ${ }^{\dagger}$ private practice, Bologna, Italy

Reprint requests: Carl E. Misch, BS, DDS, MDS, 16231 West Fourteen Mile Road, Suite 250, Birmingham, MI, USA 48025; e-mail: Info@Misch.com

○2003 BC Decker Inc
}

The ability to predictably form a direct bone-toimplant interface is a consistent treatment goal in implant dentistry. The two-stage surgical protocol established by Brånemark and colleagues ${ }^{1}$ to accom plish "osseointegration" consisted of several prerequisites, including ( 1 ) countersinking the implant below the crestal bone, (2) obtaining and maintaining a soft tissue covering over the implant for 3 to 6 months, and (3) maintaining a nonloaded implant environment for 
3 to 6 months. Following this procedure, a second-stage surgery was necessary to uncover these implants and place a prosthetic abutment. A high degree of longterm clinical rigid fixation has been reported following this protocol, in both completely and partially edentulous patients. ${ }^{2,3}$

The primary reasons cited for the submerged countersunk surgical approach to implant placement were ( 1 ) to reduce and minimize the risk of bacterial infection, (2) to prevent apical migration of the oral epithelium along with the body of the implant, and (3) to reduce and minimize the risk of early implant loading during bone remodeling.'

During the past 15 years, several authors have reported that implants may osseointegrate even though they reside above the bone and through the soft tissue during early bone remodeling. ${ }^{4-6}$ This surgical approach has been called a one-stage or nonsubmerged implant procedure, and it eliminates the second-stage implant uncovery surgery. As a result, the tissue discomfort and healing associated with second-stage surgery is eliminated for the patient, which is preferable for the patient. The clinician has reduced surgical time since uncovery and suture removal do not occur.

Immediate loading of a dental implant includes not only a nonsubmerged one-stage surgery, but also the loading of the implant with a provisional restoration at the same appointment, or shortly thereafter. Immediate loading was initially suggested for implants of reduced surface area to encourage a soft-tissue (periodontal ligament-like) interface between the implant and bone. ${ }^{7}$ These implants achieved a wide range of clinical survival. ${ }^{8-11}$ On occasion a direct bone interface could be developed, maintaining this condition for more than 20 years. ${ }^{12}$

Early studies in immediate loading, with a primary goal of a direct bone implant contact, have been proposed and have shown encouraging results. ${ }^{13,14}$ In 1990 and 1997, Schnitman and colleagues reported on immediate loading of 25 screw-shaped implants in nine completely edentulous mandibles with fixed prostheses. ${ }^{15,16}$ The implants, which were immediately loaded, were additional implants, not required for the final prosthesis. If the implants were to fail, the submerged implants would be uncovered and support the fixed prosthesis. If the immediate-loaded implants survived, they would be included in the final restoration. Schnitman and colleagues suggested this procedure be used only in the completely edentulous mandible, where moderate to abundant bone is present posterior and anterior to the foramina. Using this approach, $100 \%$ of the submerged unloaded implants survived, whereas three immediately loaded implants failed before 6 months and one implant failed 18 months post surgery, yielding an $84 \%$ survival over 9 years

In 1997 Tarnow and colleagues reported on immediate loading with a fixed prosthesis, using threaded implants in 10 consecutive completely edentulous cases over 5 years. ${ }^{17}$ Sixty-six of 69 implants were integrated in 6 mandibular and 4 maxillary completely edentulous arches (96\% survival), using a total of 10 to 13 implants in each arch for the final prosthesis. Unlike Schnitman and colleagues, Tarnow and colleagues often inserted and immediately loaded many more implants for the transitional prosthesis. The concept of immediate loading provides all the advantages of the one-stage surgical approach. In addition, implants are splinted together, which decreases the risk of overload by way of a greater surface area and improved biomechanical distribution. The patient does not need to wear a removable restoration during initial bone healing; this greatly increases comfort, psychological response, function, and stability during the transition period. ${ }^{18}$ Over the past few years, several authors have reported on immediate loading in the completely edentulous patient with 95 to $100 \%$ survival rates. ${ }^{19-23}$

To address the issues of immediate/early occlusal loading and crestal bone loss, a bone quality-based implant system (Biohorizons ${ }^{\text {(1) }}$, Maestro ${ }^{\mathrm{TM}}$ Dental Implants, Birmingham, AL, USA) was evaluated. This implant is designed to microstrain the bone within the physiologic zone of bone loading, and to help compensate for the ranges of mechanical properties found in different bone densities. ${ }^{24}$ The present article reports the 5 -year interim evaluation.

\section{MATERIALS AND METHODS}

A prospective two-center protocol of immediate implant loading study was begun in August 1996 at two different clinical centers to evaluate the clinical success of a bone density-based dental implant system. The patients' systemic conditions and the overall treatment protocol were similar to those in the prospective study already reported using the system with a conventional twostage approach. ${ }^{25}$ All patients were completely edentulous in the reported arch prior to implant insertion. 
Specifically, the effect of variables such as implant design and bone density (quality) on dental implant health were evaluated according to the Implant Quality Scale ${ }^{26,27}$ (Table 1).

This prospective study used the above scale since it includes first year bone loss results and allows a differentiation of success other than survival. The quality-ofhealth scale evaluates the health-disease continuum of an implant by using mobility, radiographic bone loss, probing depths, bleeding index, absence or presence of pain, and history of exudate to assess the status of each implant; it includes the first year of function. This report summarizes the clinical assessment and bone loss for cases with at least 1 year of follow-up after prosthesis loading.

The evaluation of the fixed prostheses in this prospective report was based on survival. Since the prostheses were not removed during the study, the implant mobility index was used only after the initial 4 to 7 months after immediate/early loading and prior to the

\section{TABLE 1 Implant Quality Scale}

Group

I. Optimum health

II. Satisfactory health

III.Compromised heatlth

IV. Clinical failure
Clinical Conditions

Treatment

Initial rigid fixation

No pain or tenderness on palpation, percussion, or function

$<1.5 \mathrm{~mm}$ crestal bone loss in first yr

$<1.0 \mathrm{~mm}$ bone loss in following 3-yr period

After first year, stable probing (sulcus) depth of $<4 \mathrm{~mm}$

No exudate history

No radiolucencies

$0-1$ bleeding index

Initial rigid fixation

No pain or tenderness on palpation, percussion, or function

$1.5-3 \mathrm{~mm}$ crestal bone loss in first yr

$<1.0 \mathrm{~mm}$ bone loss in following 3-yr period

$<4 \mathrm{~mm}$ probing depth from the original tissue thickness or

first year bone loss but stable in past 3 -year period

Past history of transient exudate

No radiolucencies

$0-1$ bleeding index (may have a transient 2 condition)

Initial rigid fixation

No pain or tenderness on palpation, percussion, or function

Slight tenderness

$<3 \mathrm{~mm}$ crestal bone loss in first yr

$<2 \mathrm{~mm}$ bone loss in following 3 -yr period but less than $1 / 2$ total bone loss of implant (implantitis)

$<5 \mathrm{~mm}$ probing depth and increasing in preceding $3 \mathrm{yr}$

History of exudate 1 to $2 \mathrm{wk}$ in preceding $3 \mathrm{yr}$

Slight radiolucency around crestal portion of implant

$1-3$ bleeding index

Any of the following conditions:

Pain on palpation, percussion, or function

$<0.5 \mathrm{~mm}$ horizontal mobility or any vertical mobility at stage II surgery

or prosthesis placement

Uncontrolled progressive bone loss

Loss of more than two walls of bone supporting the implant

Uncontrolled exudate

Generalized radiolucency

V. Absolute failure
Implant surgicaliy removed Implant exfoliated
Normal maintenance

Reduce stresses

Shorter intervals between hygiene treatments

Gingivoplasty

Reduce stresses

Drug therapy, antibiotics

Surgical reentry

Change prosthesis

Removal of implant 


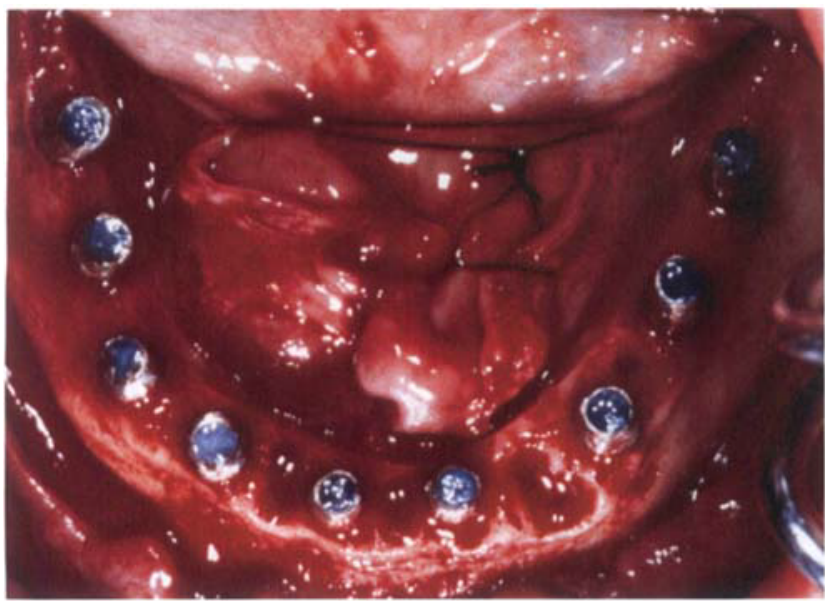

Figure 1 A mandibular arch with nine implants on the day of insertion.

delivery of the final restoration. The fixed definitive restorations were deemed successful when their service was not interrupted from the time of insertion to the time of final evaluation. If a restoration required modification or refabrication, it was noted. If a final fixed restoration was no longer possible, primarily because of implant failure, the prosthesis was deemed a failure.

\section{Study Population and Treatment}

Patients for the study were recruited in two different clinical centers. They were selected and evaluated based on medical and dental histories. Each patient signed an informed consent agreement prior to the study and was advised of the need to attend follow-up visits over a minimum period of 5 years. Disqualifying factors for the study included uncontrolled systemic conditions

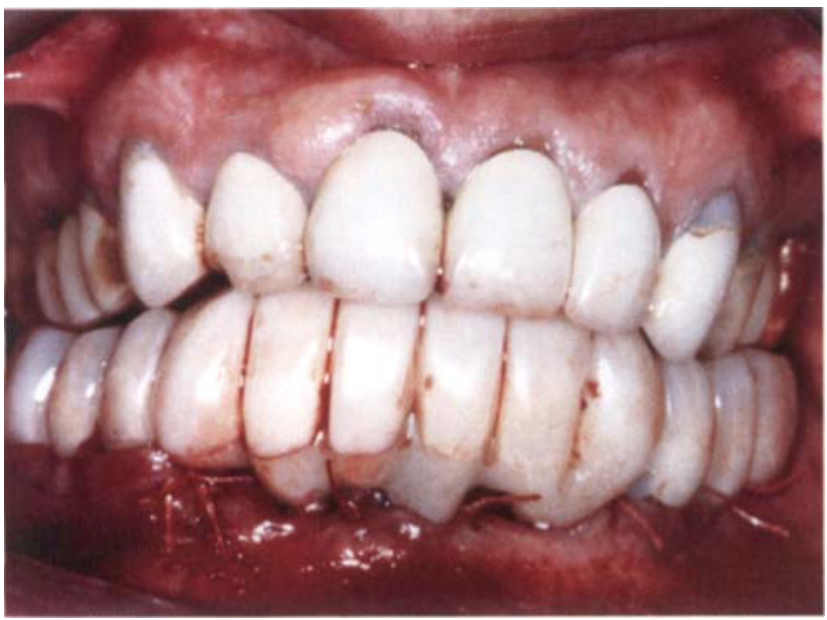

Figure 2 A prefabricated transitional prosthesis delivered on the day of surgery for the immediate occlusal load format.

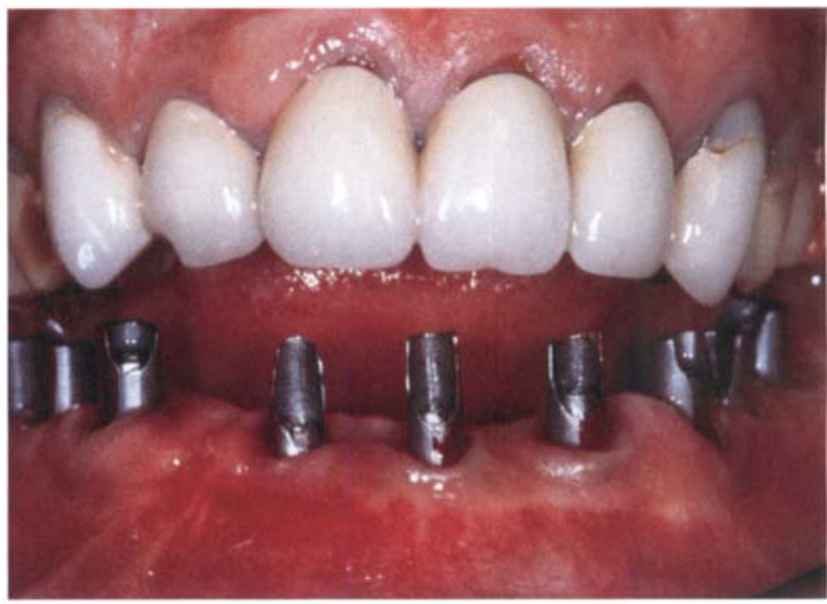

Figure 3 After 4 to 7 months' healing, the final abutments were inserted into the implant bodies.

such as severe hypertension, uncontrolled diabetes, symptomatic thyroid disorders, and pregnancy. An allograft or autograft with inadequate healing, untreated periodontitis, and inadequate oral hygiene were contraindications if present at the time of surgery.

The functional transitional prosthesis was delivered on the day of surgery or at the suture removal appointment 10 to 14 days later. When the transitional prosthesis was delivered the same day, a prefabricated restoration was relined at the conclusion of the surgery (Figures $1-4)$. When the restoration was delivered at the suture removal appointment, an implant body impression, vertical occlusal dimension, and bite registration were obtained (Figures 5-9). The abutment impression transfers were then removed and replaced with permucosal extensions, similar to the procedure in a one-stage surgi-

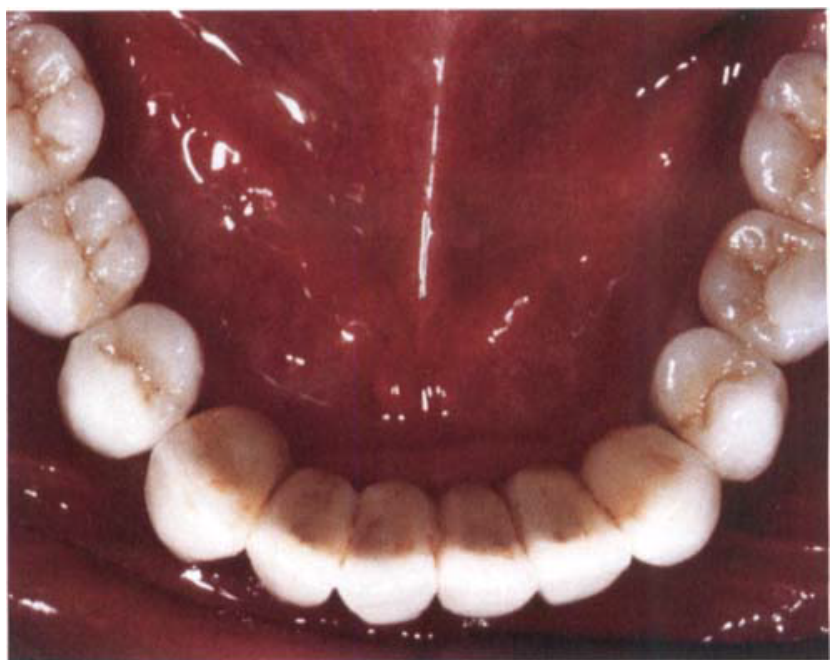

Figure 4 The final mandibular restoration in place. 


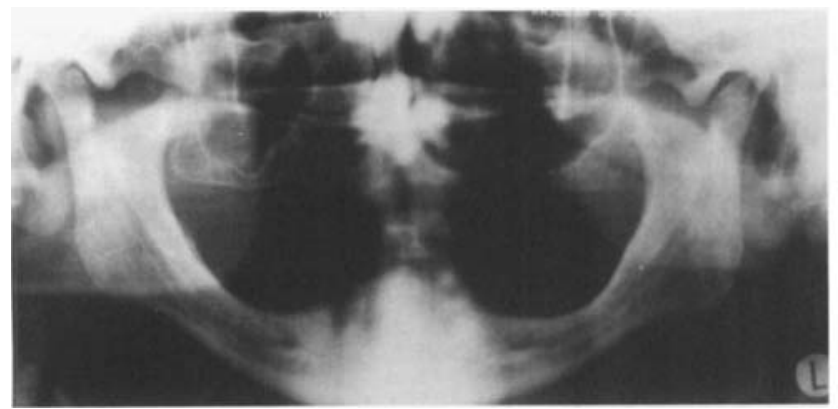

Figure 5 A preoperative radiograph of a completely edentulous patient.

cal approach (Figure 10). The laboratory modified the abutments and fabricated a transitional prosthesis (Figure 11). At the suture removal appointment, the permucosal healing abutments were removed, the abutments were inserted, and the transitional restoration was delivered (Figures 12 and 13). The final restoration was fabricated after a minimum of 4 months (Figure 14).

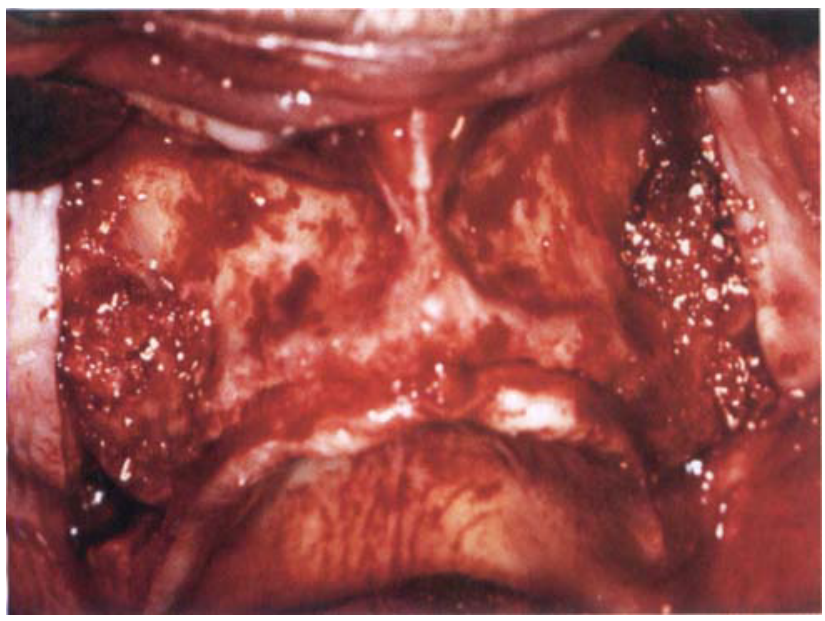

Figure 6 Bilateral sinus grafts in the maxilla and a premaxillary graft were also performed at this time.

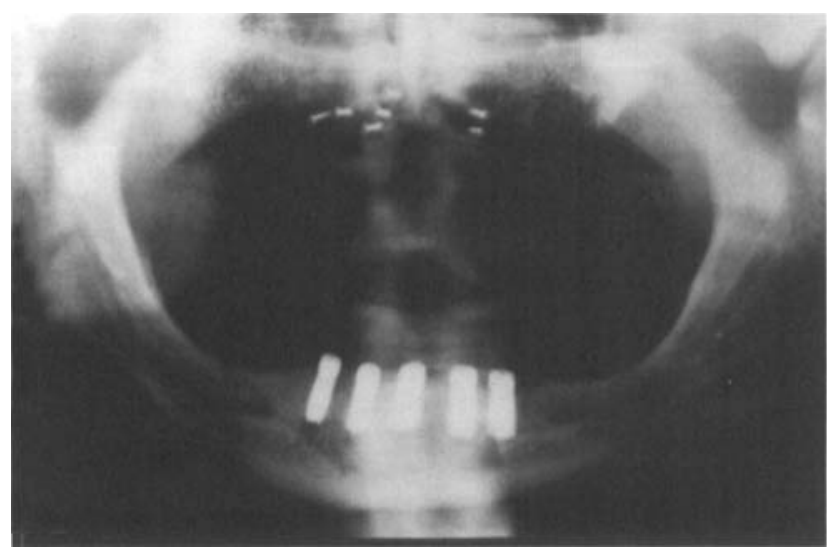

Figure 7 An immediate postoperative panoramic radiograph.

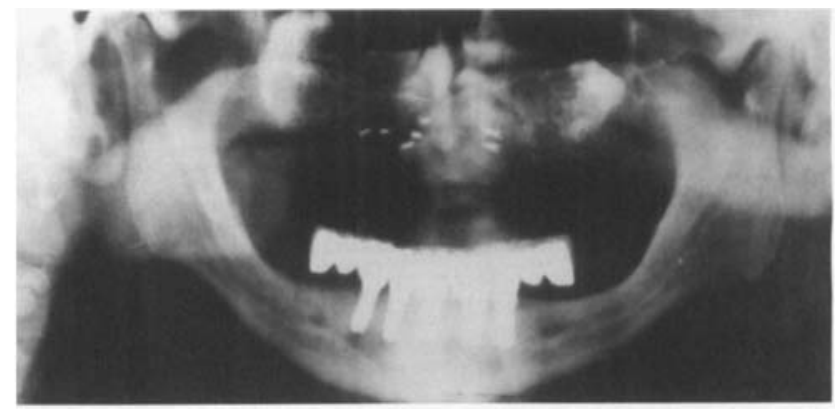

Figure 8 After 6 months the sinus grafts had matured and were radiopaque.

\section{RESULTS}

Each center was subjected to an initial audit and training by an independent study monitor (Pax Med International, San Diego, CA, USA). For purposes of this report, only cases that were a minimum of 1 year past prosthesis delivery were included in the data analysis. However, all implants placed within the report are included from the initial implant insertion. In other words, all surgical and early healing failures are reported in the data.

Intraoral radiographs were taken at the time of presurgical assessment, the initial surgery final prosthesis insertion, 6 months following prosthesis insertion, 1 year following prosthesis insertion, and yearly thereafter (Figure 15). Crestal bone remodeling was evaluated to measure bone gain or loss within $0.2 \mathrm{~mm}$ using a magnified image. The effects of any misalignment of the film plane relative to the implant axis on apparent crestal bone position were accounted for by using the known thread pitch of the implant to calibrate the measurements for each implant. The junction between the implant and the abutment (which was placed level with the bony crest at surgery) was used as a reference point to measure crestal bone changes. The difference between

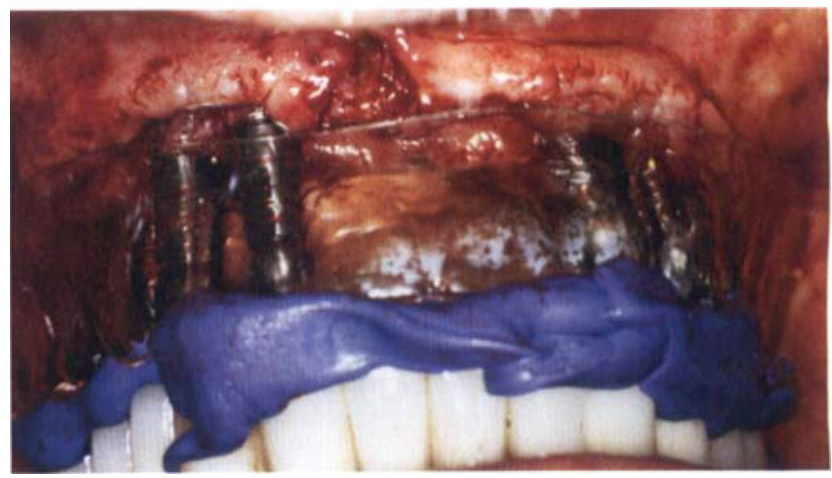

Figure 9 After implant insertion, an impression was made with a custom impression tray to transfer the vertical occlusal dimension and implant body location. 


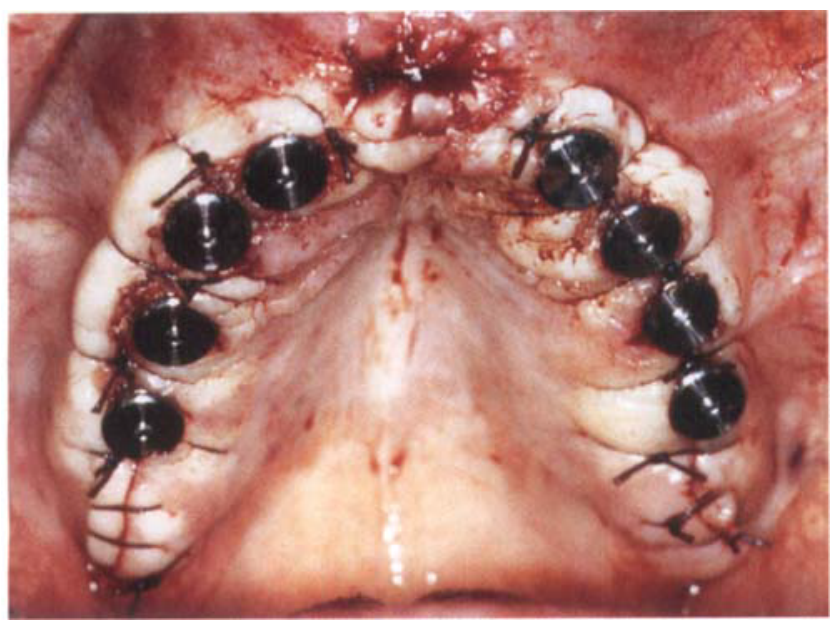

Figure 10 After the impression, the abutment transfers were removed and the permucosal extensions inserted as in a one-stage surgical approach.

mean bone level measurements at the initial surgery (assumed to be zero) and at subsequent follow-up visits was calculated and analyzed statistically. Mean cumulative bone level was calculated for each follow-up period from all readable radiographs, and interval data were calculated from the mean cumulative data (Figure 16).

The success or failure of the implant was assessed by the clinician based on the presence of persistent and irreversible pain or infection, continuous periimplant radiolucency, loss of bone support over more than onehalf of the length of the implant, or uncontrolled exudate. The range of implant success to failure was categorized as group I (optimum health) to group V (absolute failure) using the levels of health proposed by Misch $^{26,27}$ (see Table 1).

During the 5 -year period of this study, 31 arches were restored in 30 patients. There were 21 females and 9 males included, ranging in age from 39 to 84 years.

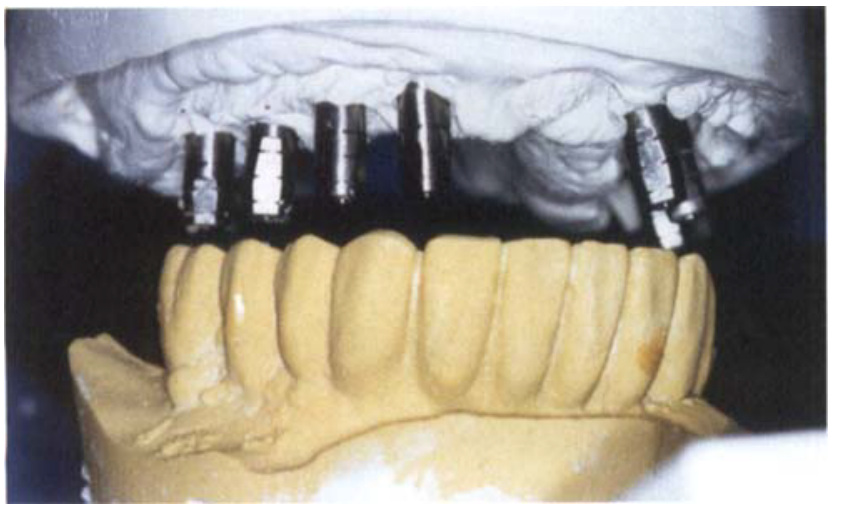

Figure 11 The laboratory poured and mounted the impression, prepared the abutments, and fabricated a transitional prosthesis.

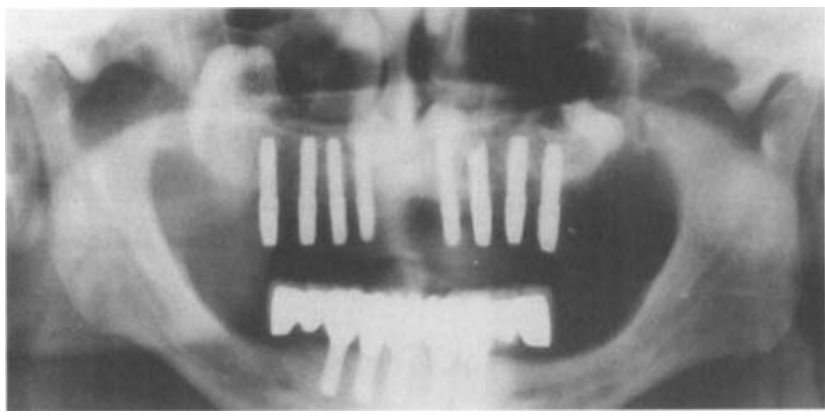

Figure 12 The abutments were inserted, and a panoramic radiograph confirmed the complete seating.

Nineteen mandibular and 12 maxillary arches were restored (both arches were involved in 1 patient); all restored arches had been completely edentulous. The mandibular immediately loaded restorations were opposed by natural dentition ( 3 patients) or complete dentures ( 16 patients). The maxillary jaws were opposed by natural dentition ( 2 patients), natural dentition and implants prostheses (2 patients), or complete implant

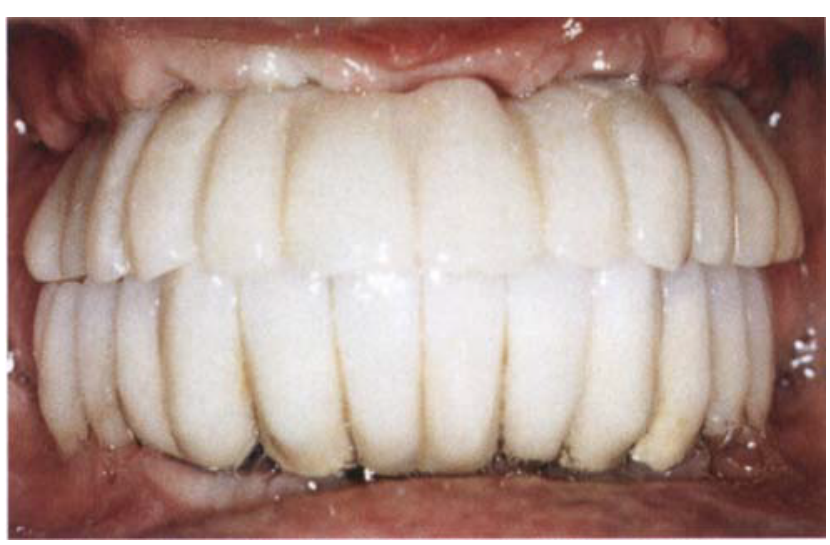

Figure 13 A fixed transitional prosthesis was delivered at the suture removal appointment, 10 to 14 days after the implant insertion surgery.

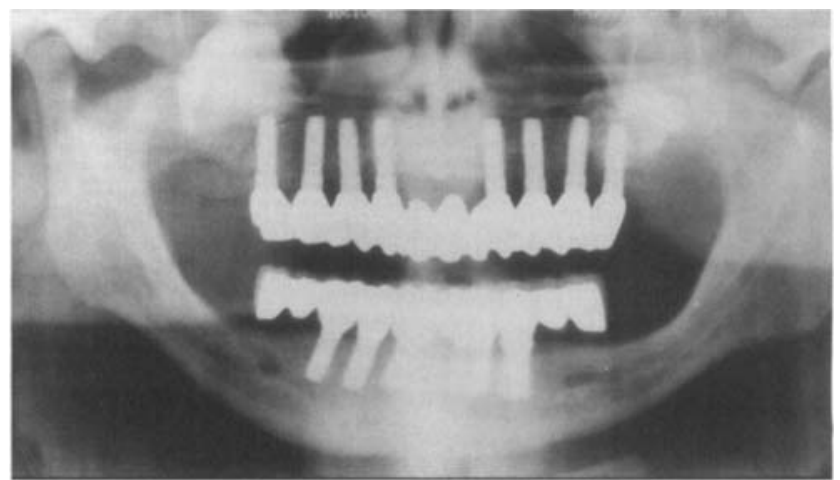

Figure 14 After 4 to 6 months, a maxillary porcelain-to-metal restoration was delivered. A panoramic radiograph confirmed complete seating. 


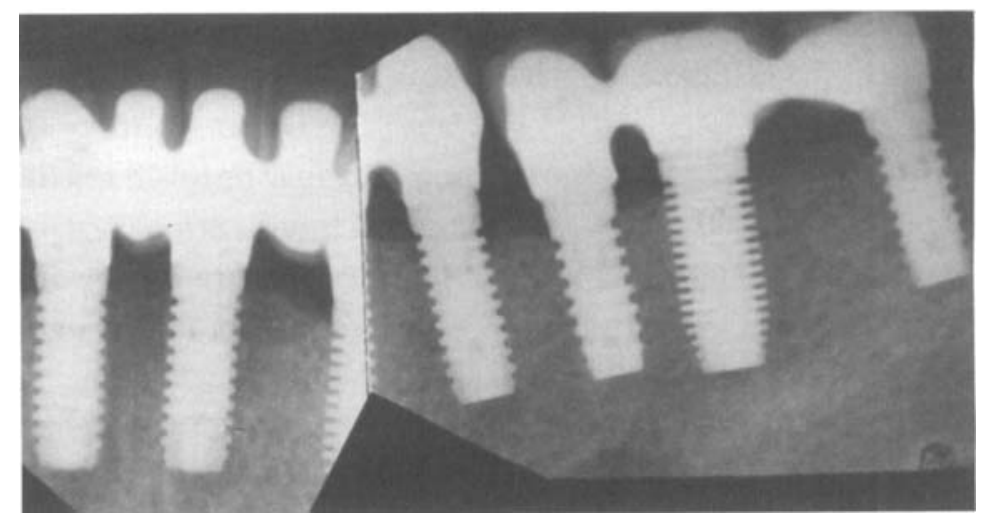

Figure 15 Periapical radiographs were obtained to evaluate crestal bone loss. The average bone loss from implant insertion to prosthesis delivery was $0.70 \mathrm{~mm}$ in both the maxilla and mandible.

prostheses ( 8 patients). A total of 244 implants were used to support these 31 restorations, for an average of 7.9 implants per prosthesis. There were 16 arches loaded the day of surgery (immediate occlusal loading) and 15 arches loaded 10 to 14 days after implant surgery (early [2 week] loading). The average maxillary prosthesis used 9 implants, whereas the average mandibular prosthesis used 7 implants.

All implants were loaded within 2 weeks of surgery, at the delivery of the transitional prostheses. The transitional prostheses had reduced occlusal load condition (ie, no cantilevers, narrow occlusal tables, acrylic occlusal surfaces). After 4 to 7 months, 30 of the final restorations were fabricated. One restoration was not finally restored for almost 2 years owing to financial reasons. There were 19 mandibular full-arch restorations, of which 14 were immediate occlusal loadings and 5 were early occlusal loadings ( 2 weeks). Of the 12 maxillary prostheses, 2 were immediate loadings and the remaining 10 were loaded at suture removal.
The bone quality-based implant system employed uses a different implant design for different bone densities. ${ }^{28}$ The hardest bone types ( 1 and 2 ) use a D2 design, whereas type 3 bone uses a D3 implant with more threads and surface area. The softest bone type (type 4) uses an implant with even more threads. The surface condition also changes for each implant design, with D2 and D3 using a resorbable blast medium (RBM) surface preparation, and the D4 implants using a hydroxyapatite surface coating. The greater surface area implant designs (D3 and D4) may also be used when load conditions are higher than usual.

The number of implants placed in the mandible ranged from 5 to 10 implants per arch, with a mode of 7 implants. The 136 implants in the mandible were primarily D3 implants, with 121 D3 implants and 15 D2 implants. There were 108 implants in the maxilla, with a range of 6 to 11 implants per arch and a mode of 8 or 9 implants. The implant design was D4 in the posterior regions (70 implants) and D3 in the anterior

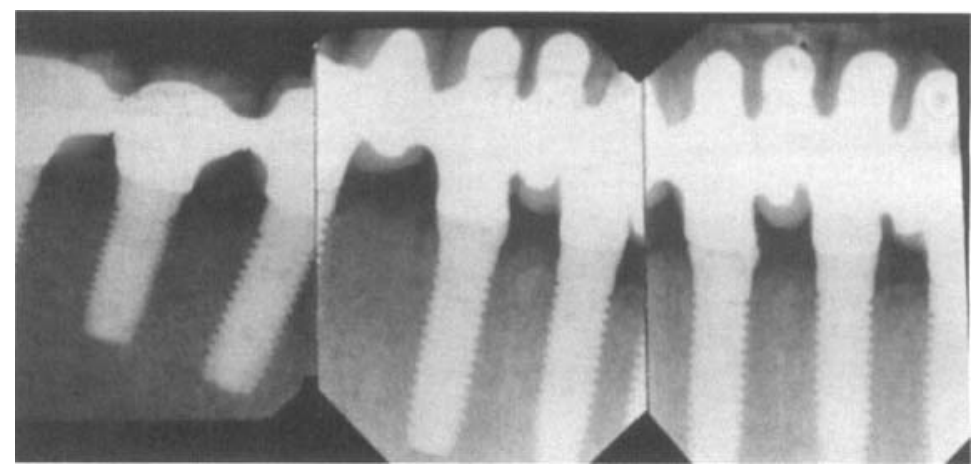

Figure 16 The first year loading periapical radiographs showed an average of $0.07 \mathrm{~mm}$ bone loss from final prosthesis delivery. After the first year of loading, a slight bone gain was observed, but the vast majority demonstrated $0 \mathrm{~mm}$ of bone loss. 
(38 implants). All implants in the maxilla were $12 \mathrm{~mm}$ long, and all but four implants in the mandible (which were $9 \mathrm{~mm}$ ) were also $12 \mathrm{~mm}$ in length.

There were no implant failures of the 244 implants followed up in this reporting period.

The prosthesis survival for 31 restorations was $100 \%$ within the time frame reported (Table 2 ). One maxillary final prosthesis was delayed for almost 2 years (22 mo) for financial considerations, prior to finishing the restoration. However, the implants remained loaded under the temporary restoration for the entire period. The follow-up period for implants and prostheses ranged from 1 to 5 years after prosthesis delivery, with an average of 2.6 years.

At the evaluation interval 1 year after prosthesis delivery, there were 229 (94\%) implants in the Misch Quality Scale group I, 9 implants (3.7\%) in group II, and 6 implants $(2.5 \%)$ in group III. At that time no implants were in groups IV or $\mathrm{V}$, which represent implant clinical or absolute failure (Table 3).

The mean overall vertical bone loss for all implants from initial surgery to prosthesis delivery was $0.70 \mathrm{~mm}$. Bone loss after the first year of prosthesis delivery has an overall mean of $0.07 \mathrm{~mm}$. A slight increase of bone was observed at the second and third years, when available to be evaluated; the vast majority demonstrated no bone loss. No significant differences were observed in bone loss among the four types of bone densities.

\section{DISCUSSION}

Cortical and trabecular bone may be modified by modeling or remodeling. ${ }^{29}$ Remodeling, or bone turnover, permits the repair of bone after trauma or allows the bone to respond to its local mechanical environment. The bone most often is lamellar, but during the repair or remodeling process it may become woven bone, which can respond more rapidly to the current situation. ${ }^{30}$ Woven bone may form at a rate of $60 \mu / d$, whereas lamellar bone forms at a rate of 1 to $5 \mu / d$. The woven bone of surgical trauma has been called repair bone; the woven bone formed from the mechanical response may be called reactive woven bone. ${ }^{31}$ When the surgical trauma is too great, or the mechanical situation is too severe, fibrous tissue may form rather than bone. Fibrous tissue at an implant interface may result with clinical mobility rather than rigid fixation.

Using the conventional healing approach, the interface bone is ready for loading at 3 to 6 months. ' Most of the surgical-related regional accelerated phenomenon (RAP) at this point is abated, and the remodeling rate owing to trauma is reduced..$^{32}$ Remodeling is also called bone turnover - it not only repairs damaged bone but also allows the implant interface to adapt to its biomechanical situation. ${ }^{33}$ The interface remodeling rate (RR) is the period of time for bone at the implant interface to be replaced with new bone. Once the bone is loaded by the implant prosthesis, the interface begins to remodel again, but this time the trigger for this process is strain, rather than the trauma of implant placement. Strain is defined as the change in length of a material divided by the original length, and it is measured as the percentage of change.

The classic two-stage surgical approach to implant dentistry permitted the surgical repair of the implant to be separated from the early loading response by 3 to 6 months. Hence, the majority of the woven bone that formed to repair the initial surgical trauma was replaced with lamellar bone. At 4 months the bone is more than $60 \%$ mineralized organized lamellar bone. ${ }^{31}$ This histologic bone type is stronger and more able to resist and/or respond to the mechanical environment of occlusal loading. ${ }^{31}$ The 4 -month healing period has been shown to be sufficient to initiate the bone-loading process in good-quality bone. One approach of implant occlusal loading is not only to reduce the risk of fibrous tissue formation, which results in clinical failure, but

TABLE 2 Summary of the Prosthesis and Implant Types and Survival

\begin{tabular}{lccccc} 
Arch & $\begin{array}{c}\text { Immediate Load } \\
\text { (Prostheses) }\end{array}$ & $\begin{array}{c}\text { Early Load (2 wk) } \\
\text { (Prostheses) }\end{array}$ & $\begin{array}{c}\text { Implant } \\
\text { Number/Type }\end{array}$ & $\begin{array}{c}\text { Survival of } \\
\text { Implants (\%) }\end{array}$ & $\begin{array}{c}\text { Survival of } \\
\text { Prostheses (\%) }\end{array}$ \\
\cline { 1 - 3 } & 2 & 10 & $108(38 \mathrm{D} 3,70 \mathrm{D} 4)$ & 100 & 100 \\
Mandible & 14 & 5 & $136(121 \mathrm{D} 3,15 \mathrm{D} 2)$ & 100 & 100 \\
Both & 16 & 15 & 244 & $100^{*}$ & $100^{\dagger}$ \\
\hline
\end{tabular}

*All implants were in Quality Scale group I to III through to last appointment.

${ }^{+}$Average follow-up was $2.6 \mathrm{yr}$ after final prosthesis delivery. 
TABLE 3 Quality Scale Groupings of Implants in Study

\begin{tabular}{cccccc} 
& Group I & Group II & Group III & Group IV & Group V \\
\hline No. of Implants (\%) & $229(94)$ & $9(3.7)$ & $6(2.5)$ & $0(0)$ & $0(0)$ \\
\hline
\end{tabular}

also to reduce the reactive woven bone formation by reducing the remodeling rate of bone necessary to repair or react to its local environment. ${ }^{24}$

\section{Immediate Occlusal Loads}

The immediate implant loading concept challenges the conventional healing time of 3 to 6 months of no loading prior to the restoration of the implant. There is generalized agreement that excess stress to an implant interface may cause overload and implant failure. ${ }^{34}$ Immediate loading of an implant does not necessarily result in excessive stress. Piatelli and colleagues ${ }^{35}$ evaluated bone reactions and the bone-titanium interface in early loaded implants in monkeys compared with unloaded implants in the same arch. No statistically significant differences were detected in the bone contact percentage after 8 months. However, loaded implants had fewer marrow spaces and more compact bone. The same group demonstrated greater bone contact in immediate-loaded implants at 9 months, ${ }^{36}$ and no fibrous tissue was found at the interface. After 15 months both unloaded and immediate-loaded implants were compared, and loaded implants exhibited greater (almost twice) direct bone contact at the interface. ${ }^{37}$ In particular, early-loaded screws demonstrated thicker lamellar and cortical bone than unloaded implants. This suggests that early occlusal loading may enhance bone remodeling and further increase bone density. Romanos and colleagues ${ }^{38}$ also demonstrated no statistical difference between immediateand delayed-loaded implants. Therefore, it appears immediate loading of an implant interface may not necessarily place the interface at increased risk of fibrous tissue formation.

The $100 \%$ survival rate of implants in this report is unusual for a clinical report of 5 years. However, this implant approach was not used as a routine methodology but was limited to abundant bone situations with more ideal crown heights. In addition, more implants and reduced force magnifiers of cantilever length were used. The traditional healing periods with this implant system have reported an implant survival of $99.4 \% .{ }^{24-39}$

\section{MICROSTRAIN REDUCTION}

One goal for an immediate-loaded implant/prosthesis system may be to decrease the risk of occlusal overload and its resultant increase in the remodeling rate of bone. Under these conditions the surgical RAP may replace the bone interface without the additional risk of biomechanical overload. When strain is placed on the horizontal axis and stress is positioned on the vertical axis, the relationship between these two mechanical indices results in the flexibility-or modulus--of elasticity of a material. Hence, the modulus conveys the amount of deformation in a material (strain) for a given load (stress) level. The lower the stress applied to the bone (force divided by the functional surface area that receives the load), the lower the microstrain in the bone. Therefore, one method to decrease microstrain and the $\mathrm{RR}$ in bone is to provide conditions that increase functional surface area to the implant bone interface.

The surface area of load may be increased in a number of ways, such as implant number, implant design, and surface conditions. A lesser force may also decrease the stress. Force may be reduced in magnitude, duration, direction, and type and may be influenced by patient conditions, implant position, and occlusal load. Hence, the number of implants per prosthesis in this report are greater than for a conventional healing approach, and implant designs provide greater surface areas, with the goal of decreasing the stress during the surgical repair phase of immediate-loaded implants.

\section{Crestal Bone Loss}

Most reports of immediate loading have evaluated implant survival only and have not addressed crestal bone loss. Crestal bone remodeling in the interval between implant placement (stage 1 surgery) and prosthesis delivery has been previously investigated in detail for the two-stage surgical approach in which the stages are separated by 3 to 6 months. Manz, in the Dental Implant Clinical Research Group (DICRG) study, revealed an overall loss of $0.94 \mathrm{~mm}$ of crestal bone with a threaded implant with an internal connection. ${ }^{40}$ Astrand and col- 
leagues observed bone losses of $1.4 \mathrm{~mm}$ and $1.8 \mathrm{~mm}$ for

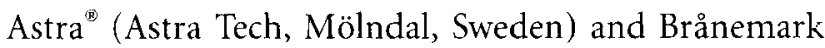
System $^{\oplus}$ implants, respectively, from the time of implant placement to prosthesis delivery 3 to 6 months later. ${ }^{41}$ Pham and colleagues found a rate of bone loss of $0.16 \mathrm{~mm}$ per month for a one-stage implant system with a traditional bone healing period. ${ }^{42}$ This corresponds to bone loss values of $0.48 \mathrm{~mm}$ to $0.96 \mathrm{~mm}$ for surgical healing periods of 3 and 6 months, respectively. Sagara and colleagues have reported greater crestal bone loss in the immediately loaded implant, compared with the twostage unloaded healing approach, in an animal study. ${ }^{43}$ It was speculated that the early loading may interfere with the ability of necrotic bone (created by the surgical trauma) to be replaced by newly formed bone. In contrast, our present study reports a mean bone loss of 0.70 $\mathrm{mm}$ between and immediate/early occlusal loading surgery and final prosthesis delivery 4 to 7 months later.

Crestal bone loss has also been addressed in the traditional-staged healing approach in the time interval between prosthesis delivery and 1-year radiographic evaluation. Astrand and colleagues reported bone losses of $0.26 \mathrm{~mm}$ and $0.17 \mathrm{~mm}$ for the Astra and Brånemark implants, respectively. ${ }^{41}$ Using an internally hexed threaded implant, the DICRG investigation reported an average bone loss of $1.14 \mathrm{~mm}$ in the same time interval. ${ }^{42}$ The present investigation of the immediate and early loading of a bone density-based system revealed an overall bone loss of $0.07 \mathrm{~mm}$ from final prosthesis delivery (4-7 mo after immediate/early loading) to 1-year radiographic evaluation.

A mean cumulative bone gain (most implants reported no change) rather than loss from 1 year or more after the first year of final prosthesis loading was reported in this investigation and is a different finding from that other systems. This unique observation may stem from the implant design. Recent human histologic data from two retrieved bone quality-based implants demonstrated that the bone turnover rate between the implant threads was less than $5 \mu / \mathrm{d}$, which corresponds to the rate of lamellar bone remodeling. ${ }^{24}$ In contrast, Garetto and colleagues ${ }^{44}$ observed reactive woven bone adjacent to conventional thread design, and bone remodeling rates 10 or more times greater. Since lamellar bone is more mineralized, rigid, and stronger than is woven bone, an implant interface composed of lamellar bone may reduce the rate of bone loss after prosthesis loading. In a histologic study, Baumgardner and col- leagues ${ }^{45}$ found that mature osteons were generally present between the threads of the bone quality-based implant system. The square thread loads the implant interface primarily in compression, which may also explain the stabilization of the bone level as well as the more rapid lamellar bone formation.

\section{Fixed versus Removable Prostheses}

Many completely edentulous patients require a removable prosthesis because of its decreased cost, personal preference, improved esthetics, or force factors. Therefore, the number of patients using the approach of immediate occlusal loading described in this article is limited. When a completely edentulous patient is a candidate for immediate loading, more implants than usual are inserted, so that the immediately load implants are not used in the final restoration, ${ }^{15,16}$ or to increase the surface area of implant support and decrease the impact and risk of implant failure. ${ }^{17}$ In either technique, more implants than usual are inserted; this increases the patient's surgical costs. As a consequence, the number of patients desiring this procedure are reduced even further.

\section{SUMMARY}

The majority of clinical reports reveal similar survival rates between immediately loaded and two-stage unloaded healing approaches in the completely edentulous patient. Nonetheless, these findings do not imply that a submerged surgical approach is no longer necessary or prudent in many cases. Future studies may find indications based on surgical-, host-, implant-, and occlusal-related conditions more beneficial for one versus the other. Higher implant failure and greater crestal bone loss seem likely in the softer bone types but, as yet, are not reported in the literature. In the current report, no implant failure occurred, and crestal bone loss values were similar to or less than values reported with the conditional two-stage approach. This may be related to the number and position of implants, implant design, and/or the surface condition of the implant loading.

\section{REFERENCES}

1. Brănemark $\mathrm{PI}$, Hansson $\mathrm{BO}$, Adell R, et al. Osseointegrated implants in the treatment of the edentulous jaw. Experience from a 10-year period. Scand J Plast Reconstr Surg 1977; 16(2 Suppl):1-132.

2. Adell R. Lekholm U, Rockler B, et al. A 15 year study of osseointegrated implants in the treatment of the edentulous jaw. Int J Oral Surg 1981; 10:387-416. 
3. Van Steenberghe D, Lekholm N, Bolender C, et al. The applicability of osseointegrated oral implants in the rehabilitation of partial edentulism; a prospective multicenter study of 558 fixtures. Int J Oral Maxillofac Implants 1990; $5: 272-281$.

4. Gotfredsen K, Hjorting-Hansen E. Histologic and histomorphometric evaluation of submerged and nonsubmerged titanium implants. In: Laney WR, Tolman DE, eds. Oral, orthopedic and maxillofacial reconstruction. Chicago: Quintessence, 1990:31-40.

5. Schroeder A, Mawglen B, Sutter F. Hohlzylinderimplantat: Typ-F zur Prothesen-retention in zahnlosen Kafer. Schweiz Monatsschr Zahnherlkunde 1983; 93:720-733.

6. Buser D, Weber HP, Bragge U, et al. Tissue integration of one stage ITI implants: 3 year results of a longitudinal study with hollow cylinder and hollow screw implants. Int J Oral Maxillofac Implants 1991; 6:405-412.

7. Strock AE, Strock M. Experimental work on a method for the replacement of missing teeth by direct implantation of a metal support into the alveolus. Am J Orthod Oral Surg 1939; 25:467.

8. Linkow LI. The blade-vent-a new dimension in endosseous implants. Dent Concepts 1968; 11:3-14.

9. Cranin AN, Rookin MF, Garfinkel L. A statistical evaluation of 952 endosteal implants in humans. J Am Dent Assoc 1977; 94:315-319.

10. Smithloff M, Fritz ME. The use of blade implants in a selected population of partially edentulous adults: a fiveyear report. J Periodontol 1976; 47:19-24.

11. Kapur KK. Veterans Administration co-operative dental implant study comparison between fixed partial dentures supported by Blade-Vent implants and partial dentures. I Prosthet Dent 1987; 59:499-512.

12. Linkow LI, Donath K, Lemons JE. Retrieval analysis of a blade implant after 231 months of clinical function. Implant Dent 1992; 1:37-43.

13. Babbush CA, Kent JN, Misiek DJ. Titanium plasma spray (TPS) screw implants for the reconstruction of the edentulous mandible. J Oral Maxillofac Surg 1986; 44:274-282.

14. Buser D, Schroeder A, Sutter F, Lang NP. The new concept of ITI hollow cylinder and hollow-screw implants. Part 2. Clinical aspects, indications, and early clinical results. Int J Oral Maxillofac Implants 1988; 3:173-181.

15. Schnitman DA, Wohrle PS, Rubenstein JE. Immediate fixed interim prostheses supported by two-stage threaded implants: methodology and results. J Oral Implantol 1990; 16:96-105.

16. Schnitman DA, Wohrle PS, Rubenstein JE, DaSilva JD, Wang NH. Brånemark implants immediately loaded with fixed prostheses at implant placement. Ten year results. Int J Oral Maxillofac Implants 1997; 12:495-503.

17. Tarnow DP, Emitiag S, Classi A. Immediate loading of threaded implants at stage one surgery in edentulous arches. Ten consecutive case reports with 1 to 5 year data. Int J Oral Maxillofac Implants 1997; 12:319-324.

18. Salama H, Rose LF, Salama M, Betts NJ. Immediate loading of bilaterally splinted titanium root-form implants in fixed prosthodontics-technique reexamined: two case reports. Int J Periodontics Restorative Dent 1995; 15:344-361.

19. Scortecci G. Anchored disk-design implants without bone augmentation in moderately to severely resorbed completely edentulous maxillae. J Oral Implant 1999; 25:37-79.

20. Randow R, Ericsson I, Nilner K, Petersson A, Glantz PO. Immediate functional loading of Brånemark dental implants. An 18-month clinical follow-up study. Clin Oral Implants Res 1999; 10:8-15.

21. Horiuchi K, Uchida $H$, Yamamoto K, Sugimura M. Immediate loading of Brånemark system implants following placement in edentulous patients: a clinical report. Int $\mathbf{J}$ Oral Maxillofac Implants 2000; 15:824-830.

22. Ganeles J, Rosenberg MM, Holt RL, Rechman LH. Immediate loading of implants with fixed restorations in the completely edentulous mandible: report of 27 patients from a private practice. Int J Oral Maxillofac Implants 2001; 16:418-426.

23. Jaffin RA, Kumar A, Berman CL. Immediate loading of implants in partially and fully edentulous jaws: a series of 27 case reports. J Periodontol 2000; 71:833-838.

24. Misch CE, Qu AL, Bidez MW. Mechanical properties of trabecular bone in the human mandible. Implications for dental implant treatment planning and surgical placement. J Oral Maxillofac Surg 1999; 57:700-706.

25. Misch CE, Dietsh-Misch F, Hoar J, et al. A bone quality-based implant system: first year of prosthetic loading. J Oral Implantol 1999; 25:185-197.

26. Misch CE. Implant success or failure: clinical assessment in implant dentistry. In: Misch CE, ed. Contemporary implant dentistry. St. Louis (MO): CV Mosby, 1993:29-42.

27. Misch CE. Implant quality scale: a clinical assessment of health-disease continuum. Oral Health 1998; July;18-26.

28. Misch CE. Density of bone: effect on treatment plans, surgical approach, healing and progressive bone loading. Int J Oral Implant 1990; 6(2):23-31.

29. Enlow DH. Principles of bone remodeling. Springfield (IL): Charles C. Thomas, 1963.

30. Roberts WE, Turkey PK, Brezniak N, Fielder PJ. Implants: bone physiology and metabolism. CDA J 1987; 15:54-61.

31. Roberts WE, Smith RK, Zilerman Y, Mozary PG, Smith RS. Osseous adaptation to continuous loading of rigid endosseous implants. Am J Orthod 1984; 86:95-111.

32. Frost HM. The regional acceleratory phenomenon: a review. Henry Ford Hosp Med Bull 1983; 31:3-9.

33. Parfitt AM. The physiological and clinical significance of bone histomorphometric data. In: Reck RR, ed. Bone histomorphometry, techniques and interpretation. Boca Raton (FL): CRC Press, 1983:143-223.

34. Brunski JB. Biomechanical factors affecting the bone-dental implant interface: review paper. Clin Mater 1992; 10:153-201.

35. Piattelli A, Corigliano M, Scarano A, Costigliola G, Paolantonio M. Immediate loading of titanium plasma-sprayed implants: an histologic analysis in monkeys. J Periodontol $1998 ; 69: 321-327$. 
36. Piattelli A, Corigliano M, Scarano A, Quaranta M. Bone reactions to early occlusal loading of two-stage titanium plasma-sprayed implants: a pilot study in monkeys. Int J Periondontics Restorative Dent 1997; 17:162-169.

37. Piattelli A, Ruggeri A, Franchi M, Romasio N, Trisi P. An histologic and histomorphic-metric study of bone reactions to unloaded and loaded non-submerged single implants in monkeys: a pilot study. J Oral Implant 1993; 19:314-320.

38. Romanos $\mathrm{G}$, Tok CG, Sias $\mathrm{CH}$, et al. Peri-implant bone reactions to immediately loaded implants. An experimental study in monkeys. J Periodontol 2001; 72:506-511.

39. Misch CE, Poitras Y, Dietsh-Misch F. Endosteal implants in the edentulous posterior maxilla-rationale and clinical report. Oral Health 2000; August:7-16.

40. Manz MC. Radiographic assessment of peri-implant vertical bone loss: DICRG interim report no. 9. J Oral Maxillofac Surg 1996; 55:621-671.
41. Astrand P, Engquist B, Dahlgren S, et al. Astra Tech and Brånemark System implants: a prospective 5-year comparative study. Results after one year. Clin Implant Dent Relat Res 1999; 1(1):17-26.

42. Pham AN, Fiorellini JP, Paquette D, et al. Longitudinal radiographic study of crestal bone levels adjacent to non-submerged dental implants. J Oral Implantol 1994; 20(1):26-34.

43. Sagara $M$, Akagawa $Y$, Nikai $H$, Tsury $H$. The effects of early occlusal loading in one-stage titanium alloy implants in beagle dogs: a pilot study. J Prosthet Dent 1993; 69:281-288.

44. Garetto LP, Chen I, Parr IA, Roberts WE. Remodeling dynamics of bone supporting rigidly fixated titanium implants: a histomorphometric comparison of four species including humans. Implant Dent 1995; 4:235-243.

45. Baumgardner JD, Boring JG, Cooper RC, et al. Preliminary evaluation of new dental implant design in canine models. Implant Dent 2000; 9:252-260. 